\title{
Memoria y metamemoria durante la amnesia global transitoria: estudio comparativo sobre su evolución a largo plazo
}

\author{
Eugenia Marín-García, José M. Ruiz-Vargas
}

Introducción. Los criterios diagnósticos de la amnesia global transitoria (AGT) establecen que el funcionamiento de la memoria se debe restablecer, como máximo, en 24 horas. Sin embargo, existen datos contradictorios sobre si aparecen secuelas a largo plazo. Además, no está claro qué herramienta es la más adecuada para controlar la evolución de estos pacientes.

Objetivos. Evaluar la afectación de la memoria episódica de los pacientes con AGT durante el episodio amnésico y a los 7 , 30 y 90 días después, con la tarea de recuerdo libre y con el test minimental, y estudiar la metamemoria de estos pacientes.

Sujetos y métodos. Se han evaluado 17 pacientes con AGT y 17 voluntarios igualados en edad, sexo y nivel educativo con el grupo de pacientes. Se ha realizado un estudio longitudinal (cuatro evaluaciones) factorial (con el grupo de pacientes y el grupo control).

Resultados. Durante la AGT, los pacientes muestran afectación de la memoria episódica tanto utilizando la tarea de recuerdo libre como el test minimental. Sin embargo, sólo la prueba de recuerdo libre detecta una mejoría a lo largo del tiempo y una puntuación alterada tres meses después. Por otra parte, se ha evidenciado que la metamemoria de estos pacientes es poco precisa.

Conclusiones. Tanto la tarea de recuerdo libre como el test minimental detectan alteración durante la AGT, pero sólo la primera es una prueba sensible a la alteración de la memoria a largo plazo asociada a este tipo de amnesia.

Palabras clave. Amnesia. Amnesia global transitoria. Memoria. Metamemoria. Minimental. Recuerdo libre.

\section{Introducción}

En el ámbito de las amnesias transitorias [1], la amnesia global transitoria (AGT) es un síndrome caracterizado por una grave amnesia anterógrada y una amnesia retrógrada de intensidad variable. Pese a la afectación de la capacidad para recordar, los pacientes mantienen sus capacidades atencionales y su conciencia preservadas [2,3].

El sistema episódico es el sistema más gravemente alterado en los pacientes con AGT [4]. La memoria episódica es declarativa, explícita y nos permite codificar, almacenar y recuperar representaciones de eventos experimentados personalmente, detallando su contexto espaciotemporal [5]. El déficit episódico más característico de los pacientes con AGT es el anterógrado, que se manifiesta por la incapacidad para adquirir información nueva y mantenerla en su memoria a largo plazo. Esto sucede con cualquier tipo de información, ya sea visual -figuras y palabras-, táctil, olfativa o auditiva -soni- dos ambientales e información verbal visual y auditiva- [6-9].

Respecto a la recuperación del episodio amnésico, los criterios diagnósticos de la AGT establecen que debe darse un restablecimiento completo en 24 horas como máximo [2]. Sin embargo, no hay datos concluyentes sobre si esto sucede así [10-15].

El hecho de que no haya conclusiones firmes sobre los posibles déficits a largo plazo después de un episodio de AGT puede deberse, en parte, a que frecuentemente se han llevado a cabo investigaciones en las que aparecían ciertos problemas metodológicos. Por ejemplo, en muchas ocasiones no se controla la variable del tiempo exacto transcurrido desde el episodio de AGT, por lo que se mezclan datos de evaluaciones post-AGT realizadas desde días hasta años después del episodio [14-17], lo que conduce a conclusiones confusas.

Además, el hecho de efectuar estudios retrospectivos hace que no se disponga de una evaluación durante la AGT, lo que impide analizar si existe me-
Departamento de Psicología Básica. Facultad de Psicología. Universidad Autónoma de Madrid. Madrid, España.

Correspondencia:

Dra. Eugenia Marín García. Departamento de Psicología Básica. Facultad de Psicología. Universidad Autónoma de Madrid. Ciudad Universitaria de Cantoblanco. Iván Pavlov, s/n. E-29049 Madrid. Fax: +34914975215.

E-mail:

eugenia.marin@uam.es

Financiación:

Beca-contrato de Formación de Personal Investigador del Gobierno Vasco.

Agradecimientos:

A los pacientes, sus familias y los voluntarios, por su colaboración. Al Servicio de Neurología de los hospitales Ramón y Cajal, La Paz y Gregorio Marañón de Madrid, en los que se realizó la detección de estos pacientes, y especialmente a los residentes de dichos servicios durante los cursos 2005-2006 y 2006-2007. Al Dr. Masjuán, por su ayuda y disposición.

Aceptado tras revisión externa: 26.01.11.

Cómo citar este artículo: Marín-García E, Ruiz-Vargas JM. Memoria y metamemoria durante la amnesia global transitoria: estudio comparativo sobre su evolución a largo plazo. Rev Neurol 2011; 53: 15-21.

(C) 2011 Revista de Neurología 
joría o no en las evaluaciones post-AGT dentro del grupo de pacientes respecto a sí mismos y no respecto al grupo control.

En relación con el modo de evaluación, en la experiencia clínica diaria, la mayoría de los clínicos se encuentra con la limitación del tiempo a la hora de evaluar a estos pacientes tanto en urgencias como en la consulta. Por tanto, muchas veces se opta por la utilización de la prueba minimental para realizar el seguimiento de los pacientes con AGT. Sin embargo, no está claro que esta prueba sea una herramienta lo suficientemente sensible como para detectar las posibles alteraciones a largo plazo de esta alteración [14].

Respecto a la metamemoria, es decir, el grado de conocimiento que se dispone sobre nuestra propia memoria y su funcionamiento [18], cabe preguntarse hasta qué punto son exactas las informaciones que los clínicos reciben por parte de los pacientes al realizar el seguimiento.

\section{Objetivo}

Los objetivos de este estudio han sido, por una parte, analizar si existen alteraciones de memoria episódica a largo plazo en pacientes que han padecido AGT y hacerlo superando los problemas metodológicos encontrados en estudios anteriores. Además, se ha estudiado qué herramienta mide con más precisión la posible alteración de memoria episódica durante la AGT y durante su recuperación, si los ítems sobre memoria (memoria inmediata y recuerdo diferido) y sobre orientación (temporal y espacial) del test minimental o la tarea de recuerdo libre, siendo ambas pruebas de rápida administración.

Por último, se ha examinado la metamemoria de los pacientes con AGT. De esta manera, se ha analizado cómo de informativa es la opinión de los pacientes sobre su estado de su memoria durante y después de la AGT.

\section{Sujetos y métodos}

\section{Muestra}

El grupo AGT está formado por los 17 pacientes (12 mujeres y 5 hombres) que durante septiembre de 2005 y julio de 2007 fueron diagnosticados de AGT por los servicios de neurología de los Hospitales Ramón y Cajal, La Paz y Gregorio Marañón de Madrid, siguiendo los criterios diagnósticos de Hodges y Warlow [2], que pudieron evaluarse durante la fase aguda de esta alteración y que hicieron las tres evaluaciones de seguimiento.

La media de edad de este grupo en el momento del episodio amnésico ha sido de 61,71 años. El 64,7\% de la muestra tiene un nivel educativo de primaria y el 35,3\% restante de estudios universitarios.

El grupo control está formado por 17 voluntarios igualados con el grupo AGT en edad (media: 61,29 años), nivel educativo (primaria, 64,7\%, y superior, 35,3\%) y sexo (12 mujeres y 5 hombres).

Ninguno de los pacientes ni de los participantes voluntarios presenta una historia de abuso de alcohol o drogas, padece epilepsia, ha sufrido un traumatismo recientemente ni está en tratamiento psiquiátrico.

\section{Procedimiento}

Durante la fase aguda de la AGT, se ha solicitado a los familiares un consentimiento informado y se ha procedido con la evaluación neuropsicológica. Después, se han realizado las sucesivas evaluaciones a la semana, al mes y a los tres meses.

\section{Diseño}

Con el fin de realizar un seguimiento de los efectos de la AGT en la memoria, se ha utilizado un diseño longitudinal factorial $(2 \times 4)$. Con una variable independiente ex post facto e intersujeto, grupo diagnóstico, con dos niveles (AGT y control); y otra variable independiente casi experimental e intrasujeto, momento de la evaluación, con cuatro niveles (durante la AGT, 7, 30 y 90 días después). La variable dependiente es el estado de la memoria obtenido mediante la evaluación con las distintas pruebas.

\section{Recuerdo libre}

En esta prueba se presenta auditivamente un conjunto de 15 palabras ordenadas aleatoriamente y se solicita a los participantes que recuerden todas las palabras escuchadas que puedan y en el orden que quieran. La puntuación de esta prueba consiste en el número de palabras correctamente recordadas.

Para la preparación de las listas de palabras, se tomó como referencia el conjunto de ítems del estudio normativo de Algarabel et al [19], y la selección de las palabras se realizó en función de los siguientes criterios restrictivos:

- Ser sustantivos de al menos dos sílabas.

- Tener valores medios en 'imaginabilidad' (rango: 4,36-5,64), 'familiaridad' (rango: 3,5-4,5), 'concreción' (rango: 3-5) y 'significatividad' (rango: 3,5- 
$4,5)$, además de la 'frecuencia de uso' entre valores de 5 y 53 (rango total: 5-941).

Esta selección se deriva de la evidencia empírica sobre los efectos de las puntuaciones extremas de estas variables sobre el nivel de aciertos y de falsas alarmas [20]. En cada una de las cuatro evaluaciones se presentaron listas de palabras diferentes, pero equiparables en los parámetros antes mencionados.

\section{Miniexamen del estado mental}

El miniexamen cognitivo [21] es una adaptación del Minimental State Examination [22]. Se trata de una prueba estándar para el examen abreviado del estado mental y para la evaluación rápida de la alteración cognitiva en la que se miden los siguientes aspectos: orientación temporal y espacial, memoria inmediata, atención y cálculo, recuerdo diferido, lenguaje y praxia constructiva elemental. Su puntuación máxima es de 30 puntos y el punto de corte para la detección de alteración es de 23/24 [23].

\section{Escala de autoevaluación sobre}

el funcionamiento de la memoria

Con esta prueba [24] se evalúa la metamemoria, es decir, el grado de conocimiento que tienen las personas sobre el funcionamiento de su propia memoria. Se dan las siguientes instrucciones: 'Ahora le voy a hacer unas preguntas sobre cómo cree que es su capacidad para recordar. Puntúe cada una de las siguientes cuestiones en función de estos criterios: 0 , muy mala; 1 , mala; 2 , regular; 3 , buena; 4 , muy buena.' La puntuación máxima es de 56 .

\section{Resultados}

Con el propósito de analizar la evolución de los pacientes con AGT, se realizaron ANOVA de medidas repetidas 2 (grupo) $\times 4$ (momento de la evaluación) para cada una de las pruebas. Para evaluar si se cumplía el supuesto de esfericidad necesario para realizar un ANOVA de medidas repetidas, se llevó a cabo la prueba de esfericidad de Mauchly. En el caso de que no se cumpliera el supuesto, se aplicó la corrección de Huynh y Feldt [25].

Como al aparecer un efecto de interacción la significación de los efectos principales puede llevar a conclusiones erróneas [26], se debe realizar el análisis de la interacción antes de sacar cualquier conclusión. Los análisis de interacción se han realizado de la siguiente manera [26,27]:
Tabla I. Estadísticos descriptivos de las puntuaciones en la tarea de recuerdo libre en las cuatro evaluaciones $(n=17)$.

\begin{tabular}{llcc}
\hline & Grupo & Media & $\begin{array}{c}\text { Desviación } \\
\text { estándar }\end{array}$ \\
\hline Evaluación 1 & AGT & 1,65 & 1,115 \\
& Control & 3,82 & 1,38 \\
\hline Evaluación 2 & AGT & 2,59 & 1,583 \\
\hline Evaluación 3 & Control & 3,88 & 1,495 \\
& AGT & 2,76 & 1,348 \\
& Control & 3,88 & 1,728 \\
Evaluación 4 & AGT & 2,94 & 1,478 \\
& Control & 4,47 & 1,772 \\
\hline
\end{tabular}

AGT: amnesia global transitoria.

- Se han examinado las diferencias entre las cuatro evaluaciones dentro de cada grupo mediante la realización de un ANOVA de medidas repetidas, pero con cada grupo por separado. Si era significativo, para saber entre qué pares de evaluaciones aparecía la significación, se realizaban comparaciones a posteriori mediante el ajuste de Bonferroni para comparaciones múltiples.

- Se examinaron las diferencias entre el grupo control y el AGT en cada una de las evaluaciones mediante la realización de ANOVA de un factor. $\mathrm{Si}$ la diferencia entre grupos era significativa, se revisaban las medias para analizar cuál de las dos era la mayor.

En todos los análisis se ha considerado que una diferencia es significativa cuando $p<0,05$, y siempre que se hable de significación se está haciendo referencia a la significación estadística.

\section{Recuerdo libre}

Los resultados evidenciaron un efecto principal de la variable 'evaluación', $F_{(3,96)}=4,763 ; p<0,005$. Las comparaciones a posteriori pusieron de manifiesto que, independientemente del grupo, aparecían diferencias significativas entre la primera y la cuarta evaluación $(p<0,01)$, siendo la cuarta en la que obtuvieron una mejor puntuación.

Igualmente, apareció un efecto principal de la variable 'grupo', $F_{(1,32)}=14,220 ; p<0,005$. Las per- 
Figura 1. Puntuaciones medias del recuerdo libre correcto en los grupos de amnesia global transitoria y control durante la amnesia global transitoria y 7, 30 y 90 días después.

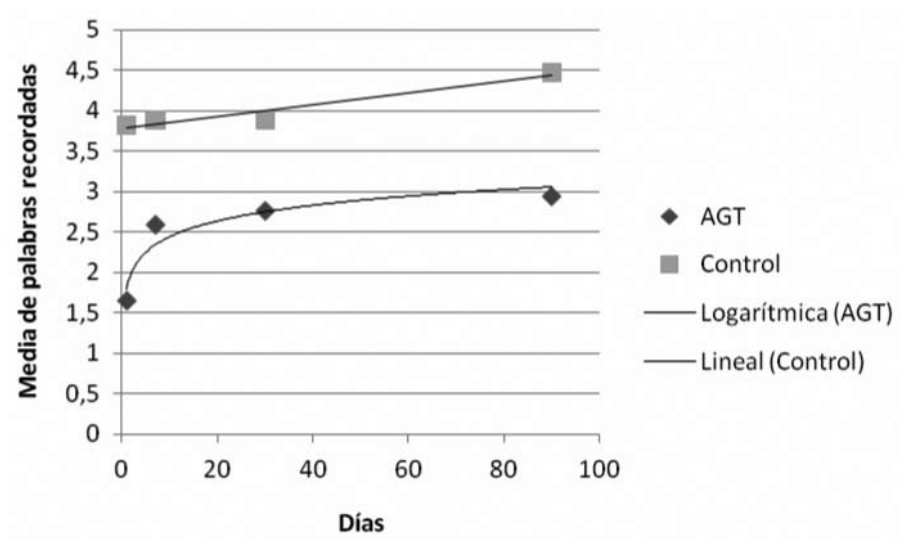

sonas del grupo control, independientemente de la evaluación, mostraban un nivel de recuerdo libre mayor que el grupo AGT (Tabla I).

Se realizó un análisis de tendencia para estudiar la evolución de la recuperación en el grupo AGT, y se obtuvo que la evolución del grupo AGT, a lo largo de los 90 días, encaja con la función logarítmica $(p<0,05)$ (Fig. 1).

Estos resultados sugieren que, con independencia de que los AGT continúen teniendo un nivel significativamente peor que el grupo control tres meses después del episodio amnésico, han experimentado una mejora significativa en la tarea de recuerdo libre. Además, esta mejoría es mucho más evidente en los primeros días desde el episodio amnésico, y después el ritmo de mejora es más lento.

La evolución del grupo control, por otra parte, encaja de forma significativa $(p<0,05)$ con una función lineal. Es probable que esta evolución se deba al aprendizaje producido al realizar la misma tarea en cuatro ocasiones.

\section{Minimental}

El ANOVA de medias repetidas evidenció efectos principales de la variable 'evaluación', $F_{(1,9,63,8)}=$ 37,$92 ; p<0,001$, y la variable 'grupo', $F_{(1,32)}=11,490$; $p<0,005$, así como la interacción 'grupo $\times$ evaluación', $F_{(1,9,63,8)}=34,982 ; p<0,001$ (Tabla II).

El análisis de la interacción reveló las siguientes conclusiones:

- Se observaron diferencias significativas entre las evaluaciones en el grupo AGT, $F_{(1,8,29,2)}=51,371$;
Tabla II. Estadísticos descriptivos de las puntuaciones totales del test minimental en las cuatro evaluaciones $(n=17)$.

\begin{tabular}{llll}
\hline & Grupo & Media & $\begin{array}{c}\text { Desviación } \\
\text { estándar }\end{array}$ \\
\hline Evaluación 1 & AGT & 24,41 & 2,181 \\
& Control & 28,65 & 1,222 \\
\hline \multirow{2}{*}{ Evaluación 2 } & AGT & 28,12 & 1,166 \\
& Control & 28,88 & 1,219 \\
\hline \multirow{2}{*}{ Evaluación 3 } & AGT & 28,94 & 0,899 \\
& Control & 28,65 & 1,412 \\
\hline \multirow{2}{*}{ Evaluación 4 } & AGT & 28,71 & 1,213 \\
& Control & 28,76 & 1,147 \\
\hline
\end{tabular}

AGT: amnesia global transitoria.

$p<0,001$. Las comparaciones a posteriori evidenciaron que las diferencias entre la primera evaluación y el resto eran significas $(p<0,001$ para todas), siendo la de la primera la más baja. Además, también aparecieron diferencias significativas entre la segunda $<$ tercera; $p<0,005$. En el grupo control no se evidenciaron diferencias significativas entre las evaluaciones.

- Las diferencias entre AGT y control fueron significativas en la primera evaluación, $F_{(1,32)}=48,575$; $p<0,001$, y marginales en la segunda, $F_{(1,32)}=3,494$; $p=0,071$. En las dos últimas no se apreciaron diferencias entre los grupos (Fig. 2).

Si se realiza el análisis por áreas, en las de orientación espacial, cálculo, memoria inmediata, lenguaje y construcción no se evidenciaron diferencias significativas ni entre los grupos ni entre las evaluaciones. Respecto a la orientación temporal, se realizó un ANOVA de medidas repetidas $2 \times 4$ en el que se evidenció la existencia de efectos principales de la variable 'evaluación', $F_{(1,6,51,8)}=30,864 ; p<0,001$, y de la variable 'grupo', $F_{(1,32)}=32,087 ; p<0,001$, así como la interacción 'grupo $\times$ evaluación', $F_{(1,6,51,8)}=$ 27,002; $p<0,001$. Y en relación con el recuerdo diferido, un ANOVA de medidas repetidas $2 \times 4$ reveló un efecto principal de la variable 'evaluación', $F_{(3,96)}=$ 9,706; $p<0,001$, así como la interacción 'grupo $\times$ evaluación', $F_{(3,96)}=10,532 ; p<0,001$.

El análisis de la interacción de ambas áreas permitió la obtención de los resultados de la tabla III. 
Estos resultados pusieron de manifiesto que el grupo AGT realizaba significativamente peor tanto la orientación temporal como el recuerdo diferido durante la primera evaluación que durante el resto de evaluaciones, mientras que el grupo control se mantenía estable a través de las evaluaciones. Por tanto, la alteración que aparece durante la AGT estaba recuperada una semana después del episodio amnésico.

\section{Escala de autoevaluación sobre}

el funcionamiento de la memoria

El ANOVA de medidas repetidas no evidenció ninguna diferencia significativa entre los grupos ni entre las evaluaciones (Fig. 3).

\section{Discusión}

El recuerdo libre de palabras está alterado durante la AGT, algo que ya se ha anotado en estudios anteriores $[7,8]$ y que es una de las características fundamentales de las amnesias crónicas [28] y del síndrome amnésico [29]. Esta alteración se debe fundamentalmente a la amnesia anterógrada que padecen los pacientes con AGT, que les impide recuperar explícitamente cualquier material nuevo y que está vinculada con una hipofunción de la región temporal, especialmente del hipocampo [30-32].

En relación con el seguimiento, en el grupo con AGT se produce una mejora a lo largo de las cuatro evaluaciones, y este progreso es mucho mayor en los primeros días posteriores al episodio amnésico, disminuyendo después la velocidad con la que mejoran. Pese a este ascenso, en todas las evaluaciones los AGT recuerdan significativamente menos palabras que los controles. Por tanto, a los tres meses de la AGT, el grupo de pacientes continúa mostrando una puntuación alterada en la prueba de recuerdo libre, lo que respalda las conclusiones de investigaciones previas que también encuentran alteración una vez superada la fase crítica. Por ejemplo, Kessler et al [13] evidencian puntuaciones anómalas en el recuerdo libre entre tres y cuatro días después de la AGT.

Una limitación de nuestro trabajo es que no queda resuelto si la alteración encontrada a los tres meses persiste en el tiempo o si se termina restableciendo completamente. A este respecto, varios estudios observan que el recuerdo libre de palabras está recuperado a largo plazo, entre cuatro y seis meses después [33] o una media de tres años después [34]. Sin embargo, otras investigaciones ponen
Figura 2. Puntuaciones medias del test minimental en los grupos de amnesia global transitoria y control durante la amnesia global transitoria, 7, 30 y 90 días después.

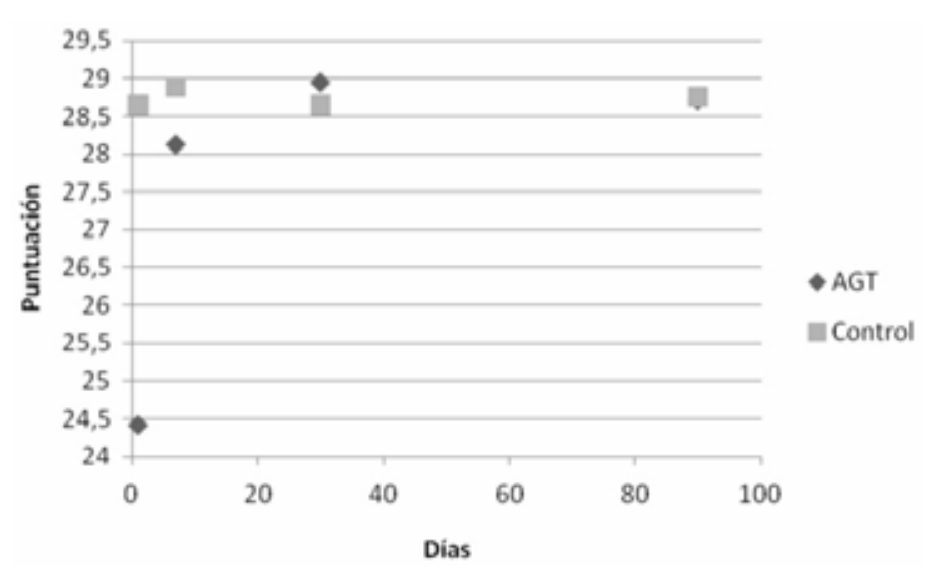

Figura 3. Puntuaciones medias de la escala de autoevaluación sobre el funcionamiento de la memoria en los grupos de amnesia global transitoria y control durante la amnesia global transitoria, 7, 30 y 90 días después.

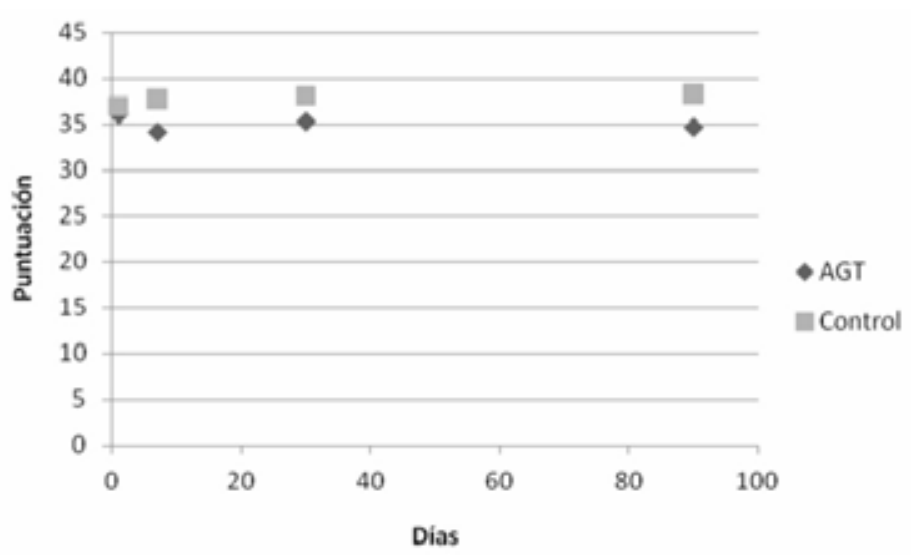

de manifiesto la alteración de la memoria verbal a largo plazo, una media de seis meses después [16] o entre uno y siete años después [13].

Por tanto, parece evidente que la memoria episódica anterógrada es la que permanece afectada durante más tiempo después de la AGT, y que la prueba de recuerdo libre es una de las más sensibles a este déficit. Pero no existe evidencia concluyente respecto a si se produce una recuperación total o no, por lo que es necesaria más investigación sobre este aspecto. 
Tabla III. Resultados del análisis de interacción para las áreas de orientación temporal y recuerdo diferido del test minimental.

\begin{tabular}{lll}
\hline & Orientación temporal & Recuerdo diferido \\
\hline & AGT: & Diferencias significativas: $F_{(1,5,24,1)}=31,838 ; p<0,001$ \\
Comparaciones a posteriori: significativas entre la primera & $\begin{array}{l}\text { AGT: } \\
\text { Diferencias significativas: } F_{(3,48)}=20,672 ; p<0,001 \\
\text { Ciferencias entre } \\
\text { evaluaciones por grupo } \\
\text { evaluación y las otras tres }(p<0,001 \text { para todas); } \\
\text { en la primera evaluación, más baja que en el resto }\end{array}$ & $\begin{array}{l}\text { primera evaluación y el resto }(p<0,001) ; \text { en la } \\
\text { primera evaluación, más baja que en el resto }\end{array}$ \\
& $\begin{array}{l}\text { Control: sin diferencias significativas entre las } \\
\text { evaluaciones }\end{array}$ & $\begin{array}{l}\text { Control: sin diferencias significativas entre las } \\
\text { evaluaciones }\end{array}$ \\
\hline \multirow{2}{*}{$\begin{array}{l}\text { Diferencias entre } \\
\text { grupos por evaluación }\end{array}$} & $\begin{array}{l}\text { Diferencias significativas sólo en la primera evaluación, } \\
F_{(1,32)}=36,994 ; p<0,001, \text { donde el grupo control } \\
\text { realizaba mejor que el de AGT }\end{array}$ & $\begin{array}{l}\text { Diferencias significativas sólo en la primera evaluación, } \\
F_{(1,32)}=28,463 ; p<0,001, \text { donde el grupo control } \\
\text { realizaba mejor que el de AGT }\end{array}$ \\
\hline
\end{tabular}

AGT: amnesia global transitoria.

En relación con el test minimental, durante la AGT los pacientes muestran una puntuación global significativamente más baja que el grupo control, pero a la semana no aparecen diferencias entre los grupos. Y respecto a sí mismos, el grupo AGT evidencia una mejoría a la semana respecto a la evaluación realizada durante el episodio amnésico, pero a partir de la semana no se detecta una evolución posterior. Por tanto, esta prueba revela la alteración que se produce durante la AGT, pero no es capaz de registrar la anomalía que se evidencia con la prueba de recuerdo libre a partir de una semana después.

$\mathrm{Si}$ analizamos el test minimental por áreas, durante la AGT se produce una alteración diferencial de las áreas referidas a la orientación temporal y al recuerdo diferido, que quedan restablecidas a la semana. Así, a la semana del episodio amnésico, tampoco los ítems específicos de orientación y memoria son lo suficientemente sensibles a los déficits de memoria que se evidencian con el uso de la prueba de recuerdo libre. Por tanto, es conveniente realizar el seguimiento con una prueba sensible y de administración rápida, como la de recuerdo libre.

El hecho de que durante la AGT no se evidencie una desorientación espacial pese a la amnesia anterógrada que padecen, probablemente se deba a que la amnesia pueda ser compensada por la realización de inferencias basadas en el contexto y por la utilización de conocimiento previo. Tampoco existen diferencias entre los grupos en ninguna evaluación en el área de memoria inmediata; por tanto, la capacidad de estos pacientes para mantener una cantidad limitada de información durante unos segundos está preservada. Esto se ha puesto de manifiesto utilizando otras pruebas de memoria a corto plazo, como la tarea de amplitud de dígitos [9-11].
Respecto al estudio de la metamemoria, pese a que el análisis de la escala de autoevaluación no fuera significativo, las puntuaciones sugieren que, en general, los controles muestran una estabilidad mayor en las puntuaciones que las del grupo AGT. Probablemente porque el grupo AGT no tenga claro cómo está el funcionamiento de su memoria después de haber padecido el episodio amnésico. Y también es llamativo que la puntuación mayor de este grupo sea durante la AGT y no después, lo que sugiere que, durante la AGT, estos pacientes no están siendo conscientes del grave déficit de memoria que están padeciendo.

En conclusión, aunque se evidencia una clara mejora a lo largo del tiempo, persisten alteraciones en la prueba de recuerdo libre tres meses después de la AGT. Esta alteración no es detectada por el test minimental, por lo que se recomienda usar una prueba más sensible, pero igualmente rápida de aplicar, como la de recuerdo libre. Además, en función de los datos obtenidos, como la información aportada por los pacientes sobre el estado de su memoria no parece ser muy precisa, no se puede considerar una fuente fiable a la hora de hacer el seguimiento.

\section{Bibliografía}

1. Naranjo-Fernández C, Arjona A, Quiroga-Subirana P, Payán-Ortiz M, Guardado-Santervás P, Serrano-Castro PJ, et al. Amnesia topográfica transitoria: descripción de una serie de ocho casos. Rev Neurol 2010; 50: 217-20.

2. Hodges J, Warlow C. Syndromes of transient amnesia: towards a classification. A study of 153 cases. J Neurol Neurosurg Psychiatry 1990; 53: 834-43.

3. Marín-García E, Ruiz-Vargas JM. Amnesia global transitoria: una revisión. I. Aspectos clínicos. Rev Neurol 2008; 46: 53-60. 4. Ruiz-Vargas JM, Marín-García E. Amnesia global transitoria: 
una revisión. II. Análisis neurocognitivo. Rev Neurol 2008; 46: 115-22.

5. Tulving E. Episodic memory: from mind to brain. Annu Rev Psychol 2002; 53: 1-25.

6. Shuttleworth E, Wise G. Transient global amnesia due to arterial embolism. Arch Neurol 1973; 29: 340-2.

7. Eustache F, Desgrandes B, Laville P, Guillery B, Lalevée C, Schaeffer $\mathrm{S}$, et al. Episodic memory in transient global amnesia: encoding, storage, or retrieval deficit? J Neurol Neurosurg Psychiatry 1999; 66: 148-54.

8. Quinette P, Guillery B, Desgrandes B, De la Sayette V, Viader F, Eustache F. Working memory and executive functions in transient global amnesia. Brain 2003; 126: 1917-34.

9. Goldenberg G, Podreka I, Pfaffelmeyer N, Wessely P, Deecke L. Thalamic ischemia in transient global amnesia: a SPECT study. Neurology 1991; 41: 1748-52.

10. Evans J, Wilson B, Wraight P, Hodges J. Neuropsychological and SPECT scan findings during and after transient global amnesia: evidence for differential impairment of remote episodic memory. J Neurol Neurosurg Psychiatry 1993; 56: 1227-30.

11. Bucuk M, Muzur A, Willheim K, Jurjevic A, Tomic Z, TuskanMohar L. Make love to forget: two cases of transient global amnesia triggered by sexual intercourse. Collegium Antropol 2004; 28: 899-905.

12. Kapur N, Abbott P, Footitt D, Millar J. Long-term perceptual priming in transient global amnesia. Brain Cogn 1996; 31: 63-74.

13. Kessler J, Markowitsch HJ, Rudolf J, Heiss WD. Continuing cognitive impairment after isolated transient global amnesia. Int J Neurosci 2001; 106: 159-68.

14. Borroni B, Agosti C, Brambilla C, Vergani V, Cottini E, Akkawi $\mathrm{N}$, et al. Is transient global amnesia a risk factor for amnestic mild cognitive impairment? J Neurol 2004; 251: 1125-7.

15. Le Pira F, Giuffrida S, Maci T, Reggio E, Zappalà G, Perciavalle V. Cognitive findings after transient global amnesia: role of prefrontal cortex. Appl Neuropsychol 2005; 12: 212-7.

16. Mazzucchi A, Moretti P, Caffarra P, Parma M. Neuropsychological functions in the follow-up of transient global amnesia. Brain 1980; 103: 161-78.

17. Cattaino G, Querin F, Pomes A, Piazza P. Transient global amnesia. Acta Neurol Scand 1984; 70: 385-90.

18. Flavell JH, Wellman H. Metamemory. In Kail R, Hagen J, eds. Perspectives on the development of memory and cognition. Hillsdale: LEA; 1977. p. 3-34.

19. Algarabel S, Ruiz JC, Sanmartín J. The University of Valencia's computerized word pool. Behav Res Methods 1988; 20: 398-403.
20. Lockhart RS. Methods of memory research. In Tulving E, Craik FIM, eds. The Oxford handbook of memory. New York: Oxford University Press; 2000. p. 45-57.

21. Lobo A, Ezquerra J, Gómez F, Seva A. El miniexamen cognoscitivo. Un test sencillo, práctico, para detectar alteraciones intelectivas en pacientes médicos. Acta Luso-Española 1979; 3: 189-202.

22. Folstein MF, Folstein SE, McHungh PH. A practical method for grading the cognitive state of patients for the clinician. J Psychiatr Res 1975; 12: 189-98.

23. Bermejo F, Díaz J, Porta-Etessam J. Cien escalas de interés en neurología. Barcelona: Prous Science; 2001.

24. Dalla-Barba G, Parlato V, Iavarone A, Boller F. Anosognosia, intrusions and 'frontal' functions in Alzheimer's disease and depression. Neuropsychologia 1995; 33: 247-59.

25. Field A. Discovering statistics using SPSS. London: Sage Publications; 2005.

26. León OG, Montero I. Cómo explicar el concepto de interacción sin estadística: análisis gráfico de todos los casos posibles en un diseño $2 \times 2$. Psicothema 2001; 13: 159-65.

27. Montero I, León OG. Triple imortal? Interacción. Consejos para su análisis, a modo de red. VII Congreso de Metodología de las Ciencias Sociales y de la Salud. Madrid, 2001.

28. Kopelman MD, Stanhope N. Anterograde and retrograde amnesia following frontal lobe, temporal lobe, or diencephalic lesions. In Squire LR, Schacter DL eds. The neuropsychology of memory. New York: Guilford Press; 2002. p. 47-60.

29. Parkin AJ, Leng NRC. Neuropsychology of the amnesic syndrome. Hove, UK: Lawrence Erlbaum; 1993.

30. Nakada T, Kwee I, Fujii Y, Knight R. High-field, T2 reversed MRI of the hippocampus in transient global amnesia. Neurology 2005; 64: 1170-4.

31. Cianfoni A, Tartaglioni T, Gaudino S, Pilato F, Saturno E, Tonali $\mathrm{P}$, et al. Hippocampal magnetic resonance imaging abnormalities in transient global amnesia. Arch Neurol 2005; 62: 1468-9.

32. Sander K, Sander D. New insights into transient global amnesia recent imaging and clinical findings. Lancet Neurol 2005; 4: 437-44.

33. Bartsch T, Alfke K, Stingele R, Rohr A, Freitag-Wolf S, Jansen $\mathrm{O}$, et al. Selective affection of hippocampal CA-I neurons in patients with transient global amnesia without long-term sequelae. Brain 2006; 129: 2874-84.

34. Uttner I, Weber, S, Freund W, Schmitz B, Ramspott M, Huber R. Transient global amnesia -full recovery without persistent cognitive impairment. Eur Neurol 2007; 58: 146-51.

\section{Memory and metamemory during transient global amnesia: a comparative study about long-term follow up}

Introduction. Diagnostic criteria of transient global amnesia (TGA) establishes that memory functioning has to be recovered in 24 hours. However, there are contradictory data about the existence of long-term sequelae. Furthermore, there is no consensus about which is the most suitable tool in order to use in the assessment of the follow up of these patients.

Aims. To assess episodic memory of TGA patients during amnesic episode and 7, 30 and 90 days after with free recall and Mini-Mental tasks. Moreover, it has been measured patient's metamemory.

Subjects and methods. 17 TGA patients and 17 healthy volunteers, matched by age, sex, and educational level were assessed. A longitudinal (four assessments) and factorial (patients and control group) study was carried out.

Results. During TGA, free recall and Mini-Mental tasks were significantly affected. However, only free recall task showed improvement along time and impairment after three months. It has been also detected that metamemory of TGA patients is imprecise.

Conclusions. Comparing with control group, both free recall and Mini-Mental tasks have significant lower levels during TGA, but only the first one is sensitive enough to show long-term disturbance associated with this amnesia.

Key words. Amnesia. Free recall task. Memory. Metamemory. Mini-Mental. Transient global amnesia. 